\title{
Multivariate Extreme Value Distributions for Random Vibration Applications
}

\author{
Sayan Gupta ${ }^{1}$ and C. S. Manohar ${ }^{2}$
}

\begin{abstract}
The problem of determining the joint probability distribution of extreme values associated with a vector of stationary Gaussian random processes is considered. A solution to this problem is developed by approximating the multivariate counting processes associated with the number of level crossings as a multivariate Poisson random process. This, in turn, leads to approximations to the multivariate probability distributions for the first passage times and extreme values over a given duration. It is shown that the multivariate extreme value distribution has Gumbel marginal and the first passage time has exponential marginal. The acceptability of the solutions developed is examined by performing simulation studies on bivariate Gaussian random processes. Illustrative examples include a discussion on the response analysis of a two span bridge subjected to spatially varying random earthquake support motions.
\end{abstract}

CE Database subject headings: Random vibration; Earthquakes; Random processes; Probability distribution.

\section{Introduction}

The study of probability distribution of first passage times and extreme values of random processes lies at the heart of the subject of random vibrations. These studies find extensive applications in the development of response spectrum based methods in earthquake engineering, gust factor approach in wind engineering, and in reliability analysis of random dynamical systems in general. In the context of Gaussian random processes, one of the commonly used approaches to study first passage failures is based on the assumption that the number of times a specified level is crossed can be modeled as a Poisson counting process. This leads to exponential models for the first passage times and Gumbel models for the extremes over a specified duration. Results based on this assumption, and further refinements to this approach, are widely discussed in many standard random vibration textbooks: see, for example, the books by Lin (1967); Nigam (1983); and Lutes and Sarkani (1997). An alternative approach, that is applicable to random processes that possess Markov property, has also been developed. Here, one studies the backward Kolmogorov equation governing the transition probability density function and the associated generalized Pontriagin-Vitt (GPV) equations governing the moments of the first passage times: see the paper by Roberts (1986) for a review of literature on this topic. Attempts to solve backward Kolmogorov equations and the GPV equations using the finite-element method have also been reported (Spencer and

${ }^{1}$ Research Student, Dept. of Civil Engineering, Indian Institute of Science, Bangalore 560012, India. E-mail: sayan@civil.iisc.ernet.in

${ }^{2}$ Associate Professor, Dept. of Civil Engineering, Indian Institute of Science, Bangalore 560012, India (corresponding author). E-mail: manohar@civil.iisc.ernet.in

Note. Associate Editor: Gerhart I. Schueller. Discussion open until December 1, 2005. Separate discussions must be submitted for individual papers. To extend the closing date by one month, a written request must be filed with the ASCE Managing Editor. The manuscript for this paper was submitted for review and possible publication on March 2, 2004; approved on October 20, 2004. This paper is part of the Journal of Engineering Mechanics, Vol. 131, No. 7, July 1, 2005. CASCE, ISSN 0733-9399/2005/7-712-720/\$25.00.
Bergman 1985). In the context of reliability analysis of dynamical systems, the determination of the probability distribution of extreme values enables the treatment of a time-variant reliability problem as a problem in time-invariant reliability analysis (Madsen et al. 1986; Melchers 1999). The underlying principle of this approach lies in the fact that the distribution of the extreme values of random processes over a specified duration of time is closely related to their mean level crossing rates. Outcrossing rates of vector random processes have been studied in the context of problems in load combinations (Naess 1989) and in structural reliability (Veneziano et al. 1977; Ditlevsen 1984; Wen and Chen 1989; Hagen and Tvedt 1991; Hagen 1992). The focus of many of these studies has been in determining the probability of exceedance of the sum of the component processes, and the outcrossing event has been formulated as a scalar process outcrossing. The present paper, however, addresses the problem of determining the joint probability distribution function (PDF) of the extremes of the component processes. In this context, it is of interest to note that, in the mathematical literature, the problem of extreme value distributions has received wide research attention: see, for example, the books by Galambos (1978); Castillo (1988); and Kotz and Nadarajah (2000). The focus of these studies has been on developing asymptotic forms of extreme value distributions for a sequence of scalar and vector random variables. In the latter case, one often considers a sequence of random variables, such as $\left\{X_{i}\right\}_{i=1}^{k}$ and $\left\{Y_{i}\right\}_{i=1}^{k}$, such that $X_{i}$ s are identical and independently distributed (i.i.d.), $Y_{i}$ s are i.i.d., and $X_{i}$ being correlated to $Y_{i}$. Questions on joint probability distribution of $X_{m}=\max _{1 \leqslant i \leqslant k}\left(X_{i}\right)$ and $Y_{m}=\max _{1 \leqslant i \leqslant k}\left(Y_{i}\right)$ are asked and specifically, conditions under which $X_{m}$ and $Y_{m}$ are mutually independent have been studied. As one might expect, the studies on sequence of vector random variables are relatively of recent origin and are less straightforward. These books also contain extensive surveys of related literature from mathematical statistics and probability.

A knowledge of the joint PDF of extreme values associated with a vector of mutually correlated stationary Gaussian random processes is necessary for the reliability analysis of structural systems, characterized by multiple limit states in series configurations. Leira $(1994,2003)$ developed multivariate extreme value 
distributions for a vector of Gaussian/non-Gaussian random processes, using a geometric approach based on directional extremes. In this paper, however, the development of multivariate extreme value distributions is based on the use of multipoint random processes to model the level crossing statistics of the vector parent process. Based on this, the joint distribution of first passage times is derived which, in turn, is further applied to derive the multivariate extreme value distributions. Specifically, it is shown that, if the level crossings of the vector random process are modeled as a multivariate Poisson process, the vectors of extreme values turn out to be mutually dependent random variables with Gumbel marginals. The mutual dependency characteristics here are related to the mutual cross correlations that exist among the components of the parent vector Gaussian random process. The theoretical predictions on multivariate distribution of first passage times and extreme values for bivariate Gaussian random processes are compared with corresponding results from Monte Carlo simulations. The illustrative examples include the study of an idealized model of a two-span bridge structure subjected to differential random earthquake loads.

\section{Problem Statement}

Consider $\left\{X_{i}(t)\right\}_{i=1}^{k}$ to be a vector of zero-mean, stationary, Gaussian random processes with a specified power spectral density (PSD) matrix $\mathbf{S}(\omega)$ and covariance matrix $\mathbf{R}(\tau)$. Associated with each of $X_{i}(t)$, we define $N_{i}\left(\alpha_{i}, 0, T\right)$ to be the number of times a level $\alpha_{i}$ is crossed in the interval 0 to $T, T_{f_{i}}\left(\alpha_{i}\right)$ to be the time required for $X_{i}(t)$ to cross level $\alpha_{i}$ for the first time, and $X_{m_{i}}$ $=\max _{0 \leqslant t \leqslant T} X_{i}(t)$ to be the maximum of $X_{i}(t)$ in the time interval 0 to $T$. Clearly, for a given $i, N_{i}\left(\alpha_{i}, 0, T\right), T_{f_{i}}\left(\alpha_{i}\right)$, and $X_{m_{i}}$ are all random variables. As is well-known, one class of acceptable approximations for $N_{i}\left(\alpha_{i}, 0, T\right), T_{f_{i}}\left(\alpha_{i}\right)$, and $X_{m_{i}}$ hypothesize that, for a given $i, N_{i}\left(\alpha_{i}, 0, T\right)$ is a Poisson random variable, $T_{f_{i}}\left(\alpha_{i}\right)$ is an exponential random variable, and $X_{m_{i}}$ is a Gumbel random variable (Lin 1967; Nigam 1983; Lutes and Sarkani 1997). For the vector random process $\left\{X_{i}(t)\right\}_{i=1}^{k}$, it is clear that $\mathbf{N}=\left\{N_{i}\left(\alpha_{i}, 0, T\right)\right\}_{i=1}^{k}, \mathbf{T}_{f}=\left\{T_{f_{i}}\left(\alpha_{i}\right)\right\}_{i=1}^{k}$, and $\mathbf{X}_{m}=\left\{X_{m_{i}}\right\}_{i=1}^{k}$ are all vectors of random variables. The problem on hand consists of determining the joint PDFs $P_{\mathbf{N}}(\widetilde{n}), P_{\mathbf{T}_{f}}(\widetilde{t})$, and $P_{\mathbf{X}_{m}}(\tilde{x})$ for $\mathbf{N}, \mathbf{T}_{f}$, and $\mathbf{X}_{m}$, respectively. We propose to develop these joint PDFs by generalizing the classical Poisson approximation used for modeling level crossings of scalar Gaussian random processes.

\section{Approximation for Number of Level Crossings}

Here, we hypothesize that $\left\{N_{i}\left(\alpha_{i}, 0, T\right)\right\}_{i=1}^{k}$ constitutes a vector of multivariate Poisson random variables. To clarify this notion, we begin by considering the case of $k=2$. For this case, we define three mutually independent Poisson random variables $\left\{U_{i}\right\}_{i=1}^{3}$ with parameters $\lambda_{1}, \lambda_{2}$, and $\lambda_{3}$, respectively. Introducing the transformations

$$
\begin{aligned}
& N_{1}\left(\alpha_{1}, 0, T\right)=U_{1}+U_{3} \\
& N_{2}\left(\alpha_{2}, 0, T\right)=U_{2}+U_{3}
\end{aligned}
$$

it can be shown that $N_{1}\left(\alpha_{1}, 0, t\right)$ and $N_{2}\left(\alpha_{2}, 0, T\right)$ are Poisson random variables with parameters $\left(\lambda_{1}+\lambda_{3}\right)$ and $\left(\lambda_{2}+\lambda_{3}\right)$, respectively. It may be noted that this construct for multivariate Poisson random variables has been discussed in the existing literature; see
Johnson and Kotz (1969) for these details and also for a list of references to the original work. In the context of the present study, it is important to note that the parameters $\lambda_{1}, \lambda_{2}$, and $\lambda_{3}$ are, as yet, unknowns to be determined. It can also be shown that the covariance of $N_{1}\left(\alpha_{1}, 0, T\right)$ and $N_{2}\left(\alpha_{2}, 0, T\right)$ is equal to $\lambda_{3}$. Based on this, we get the equation

$$
\left[\begin{array}{lll}
1 & 0 & 1 \\
0 & 1 & 1 \\
0 & 0 & 1
\end{array}\right]\left\{\begin{array}{c}
\lambda_{1} \\
\lambda_{2} \\
\lambda_{3}
\end{array}\right\}=\left\{\begin{array}{c}
\left\langle N_{1}\left(\alpha_{1}, 0, T\right)\right\rangle \\
\left\langle N_{2}\left(\alpha_{2}, 0, T\right)\right\rangle \\
\operatorname{Cov}\left[N_{1}\left(\alpha_{1}, 0, T\right), N_{2}\left(\alpha_{2}, 0, T\right)\right]
\end{array}\right\}
$$

Here $\langle\cdot\rangle$ denotes the mathematical expectation operator. It is well known that the counting process $N_{i}\left(\alpha_{i}, 0, T\right)$ is related to the parent process $X_{i}(t)$ through the relation (Nigam 1983)

$$
N_{i}\left(\alpha_{i}, 0, T\right)=\int_{0}^{T} \delta\left[X_{i}(t)-\alpha_{i}\right] \dot{X}_{i}(t) U\left[\dot{X}_{i}(t)\right] d t
$$

Here, $U(\cdot)$ is the Heaviside function. From Eq. (3), one gets

$$
\left\langle N_{i}\left(\alpha_{i}, 0, T\right)\right\rangle=T /(2 \pi)\left(\lambda_{2_{i}} / \lambda_{0_{i}}\right)^{0.5} \exp \left[-0.5 \alpha_{i}^{2} / \lambda_{0_{i}}\right]
$$

where $\lambda_{2}$ and $\lambda_{0_{i}}=$ spectral spectral moments given by

$$
\lambda_{j_{i}}=\int_{-\infty}^{\infty} \omega^{j} S_{i i}(\omega) d \omega, \quad j=0,1,2
$$

Here, $S_{i i}(\omega)$ is the auto-PSD function of $X_{i}(t)$. Using a similar counting procedure, as defined in Eq. (3), one can also derive

$$
\begin{aligned}
\operatorname{Cov} & {\left[N_{1}, N_{2}\right] } \\
& =\left\langle N_{1}\left(\alpha_{1}, 0, T\right) N_{2}\left(\alpha_{2}, 0, T\right)\right\rangle-\left\langle N_{1}\left(\alpha_{1}, 0, T\right)\right\rangle\left\langle N_{2}\left(\alpha_{2}, 0, T\right)\right\rangle
\end{aligned}
$$

where, $\operatorname{Cov}[\cdot]$ denotes the covariance function. The details of this derivation have been provided in Appendix I.

To construct the bivariate PDF of $N_{1}\left(\alpha_{1}, 0, T\right)$ and $N_{2}\left(\alpha_{2}, 0, T\right)$, we first write the characteristic function of $N_{1}\left(\alpha_{1}, 0, T\right)$ and $N_{2}\left(\alpha_{2}, 0, T\right)$ as

$$
\begin{aligned}
\Phi_{12}\left(\omega_{1}, \omega_{2}\right) & =\left\langle\exp \left[i \omega_{1} N_{1}+i \omega_{2} N_{2}\right]\right\rangle \\
& =\left\langle\exp \left[i \omega_{1}\left(U_{1}+U_{3}\right)+i \omega_{2}\left(U_{2}+U_{3}\right)\right]\right\rangle \\
& =\left\langle\exp \left[i \omega_{1} U_{1}\right]\right\rangle\left\langle\exp \left[i \omega_{2} U_{2}\right]\right\rangle\left\langle\exp \left[i\left(\omega_{1}+\omega_{2}\right) U_{3}\right]\right\rangle
\end{aligned}
$$

For a Poisson random variable $U_{i}$, with parameter $\lambda_{i}$, it can be shown that $\left\langle\exp \left[i \omega U_{i}\right]\right\rangle=\exp \left[-\lambda_{i}\right] \exp \left[\lambda_{i} \exp \{i \omega\}\right]$ (Srinivasan and Mehata 1976). Thus Eq. (6) can be written as

$$
\begin{aligned}
\Phi_{12}\left(\omega_{1}, \omega_{2}\right)= & \exp \left[-\left(\lambda_{1}+\lambda_{2}+\lambda_{3}\right)+\lambda_{1} \exp \left(i \omega_{1}\right)+\lambda_{2} \exp \left(i \omega_{2}\right)\right. \\
& \left.+\lambda_{3} \exp \left\{i\left(\omega_{1}+\omega_{2}\right)\right\}\right]
\end{aligned}
$$

In the present study, we accept this condition as the definition of a bivariate Poisson process. The associated bivariate Poisson probability mass function can be shown (Johnson and Kotz 1969) to be given by

$$
\begin{aligned}
& P\left[\left(N_{1}=j\right) \cap\left(N_{2}=l\right)\right] \\
& \quad=1 /\left(4 \pi^{2}\right) \int_{-\pi}^{\pi} \int_{-\pi}^{\pi} \Phi_{12}\left(\omega_{1}, \omega_{2}\right) \exp \left[-i\left(\omega_{1} j+\omega_{2} l\right)\right] d \omega_{1} d \omega_{2} \\
& \quad=\exp \left[-\left(\lambda_{1}+\lambda_{2}+\lambda_{3}\right)\right] \sum_{i=0}^{\min (j, l)}\left(\lambda_{1}^{(j-i)} \lambda_{2}^{(l-i)} \lambda_{3}^{i}\right) /\{(j-i) !(l-i) ! i !\}
\end{aligned}
$$

It is to be noted that, as one might expect, $N_{1}\left(\alpha_{1}, 0, T\right)$ and 
$N_{2}\left(\alpha_{2}, 0, T\right)$ here, have Poisson marginals. For $j=0$ and $l=0$, one gets

$$
P\left[\left(N_{1}=0\right) \cap\left(N_{2}=0\right)\right]=\exp \left[-\left(\lambda_{1}+\lambda_{2}+\lambda_{3}\right)\right]
$$

The above result can easily be generalized for the case $k>2$. The number of mutually independent Poisson random variables can be generalized to be given by $C_{1}^{k}+C_{2}^{k}$, where $C_{n}^{k}$ denotes combination of $k$ random variables taken $n$ at a time. Thus, for $k=3$, consider six mutually independent Poisson random variables $\left\{U_{i}\right\}_{i=1}^{6}$ with parameters $\left\{\lambda_{i}\right\}_{i=1}^{6}$ and define

$$
\begin{aligned}
& N_{1}\left(\alpha_{1}, 0, T\right)=U_{1}+U_{4}+U_{5} \\
& N_{2}\left(\alpha_{2}, 0, T\right)=U_{2}+U_{4}+U_{6} \\
& N_{3}\left(\alpha_{3}, 0, T\right)=U_{3}+U_{5}+U_{6}
\end{aligned}
$$

The equations relating $\left\{\lambda_{i}\right\}_{i=1}^{6}$ to the moments of $\left\{N_{i}\left(\alpha_{i}, 0, T\right)\right\}_{i=1}^{3}$ can be shown to be given by

$$
\left[\begin{array}{cccccc}
1 & 0 & 0 & 1 & 1 & 0 \\
0 & 1 & 0 & 1 & 0 & 1 \\
0 & 0 & 1 & 0 & 1 & 1 \\
0 & 0 & 0 & 1 & 0 & 0 \\
0 & 0 & 0 & 0 & 1 & 0 \\
0 & 0 & 0 & 0 & 0 & 1
\end{array}\right]\left\{\begin{array}{c}
\lambda_{1} \\
\lambda_{2} \\
\lambda_{3} \\
\lambda_{4} \\
\lambda_{5} \\
\lambda_{6}
\end{array}\right\}=\left\{\begin{array}{c}
\left\langle N_{1}\left(\alpha_{1}, 0, T\right)\right\rangle \\
\left\langle N_{2}\left(\alpha_{2}, 0, T\right)\right\rangle \\
\left\langle N_{3}\left(\alpha_{3}, 0, T\right)\right\rangle \\
\operatorname{Cov}\left[N_{1}\left(\alpha_{1}, 0, T\right), N_{2}\left(\alpha_{2}, 0, T\right)\right] \\
\operatorname{Cov}\left[N_{1}\left(\alpha_{1}, 0, T\right), N_{3}\left(\alpha_{3}, 0, T\right)\right] \\
\operatorname{Cov}\left[N_{2}\left(\alpha_{2}, 0, T\right), N_{3}\left(\alpha_{3}, 0, T\right)\right]
\end{array}\right\}
$$

As in the case of $k=2$, one can show that the joint characteristic function of $N_{1}, N_{2}$, and $N_{3}$ is given by

$$
\begin{aligned}
& \Phi_{123}\left(\omega_{1}, \omega_{2}, \omega_{3}\right) \\
& =\exp \left[-\sum_{j=1}^{6} \lambda_{j}+\sum_{j=1}^{3} \lambda_{j} \exp \left(i \omega_{j}\right)+\lambda_{4} \exp \left\{i\left(\omega_{1}+\omega_{2}+\omega_{3}\right)\right\}\right. \\
& \left.\quad+\lambda_{5} \exp \left\{i\left(\omega_{1}+\omega_{3}\right)\right\}+\lambda_{6} \exp \left\{i\left(\omega_{2}+\omega_{3}\right)\right\}\right]
\end{aligned}
$$

from which, it can further be shown that

$$
P\left[N_{1}=0 \cap N_{2}=0 \cap N_{3}=0\right]=\exp \left[-\sum_{j=1}^{6} \lambda_{j}\right]
$$

It is to be noted that for $k>2$, the formulation requires the numerical evaluation of a set of double integrals (see Appendix I) and at no stage does the order of the integrals to be evaluated become more than two. In general, the number of such integrals that need to be evaluated is $C_{2}^{k}$ for single sided thresholds and $4 C_{2}^{k}$ when two sided thresholds [of the type $-\alpha_{i} \leqslant X_{i}(t) \leqslant \alpha_{i}$ ] are considered (see Appendix I).

It must be noted, however, that there is a limitation on the applicability of the joint multivariate Poisson distribution (Johnson and Kotz 1969). For example, for the applicability of a bivariate Poisson distribution for two Poisson distributed random variables $N_{1}$ and $N_{2}$

$$
\begin{aligned}
\operatorname{corr}\left[N_{1}, N_{2}\right] & =\lambda_{3}\left(\lambda_{3}+\lambda_{1}\right)^{-1 / 2}\left(\lambda_{3}+\lambda_{2}\right)^{-1 / 2} \\
& \leqslant\left|\lambda_{3}\left\{\lambda_{3}+\min \left(\lambda_{1}, \lambda_{2}\right)\right\}^{-1}\right|
\end{aligned}
$$

where $\lambda_{i}=$ parameters of the three mutually independent Poisson random variables $\left\{U_{i}\right\}_{i=1}^{3}$, defined in Eq. (1) and corr $[\cdot]$ denotes correlation coefficient. However, in this study, we adopt an inverse approach whereby the parameters $\lambda_{i}$ are determined from the assumption that the multivariate Poisson distribution model is valid. Thus the condition in Eq. (14) is always satisfied. This has been illustrated further in the numerical examples to follow. Furthermore, it must be emphasized in this context that the assumption that the number of level crossings being Poisson distributed is not always valid. Cramer (1966) has shown that this assumption is asymptotically exact when the threshold levels increase to infinity. However, as pointed out by Vanmarcke (1975), for barrier levels of practical interest, this assumption results in errors whose size and effect depend strongly on the bandwidth of the processes (Vanmarcke 1972). For wide-band processes, the Poisson approximation makes no allowance for the time the processes spend above the threshold levels while for narrow-band processes the level crossings tend to occur in clumps. The multivariate models for joint level crossings, extreme value distributions, and first passage times developed in this paper are thus not free of these limitations.

\section{Approximation for the First Passage Time}

The probability distribution of the first passage times for a vector random process is related to the probability of the number of level crossings being zero and is given by the relation

$$
P\left[\bigcap_{i=1}^{k} T_{f_{i}}\left(\alpha_{i}\right)>t_{i}\right]=P\left[\bigcap_{i=1}^{k}\left\{N_{i}\left(\alpha_{i}, 0, t_{i}\right)=0\right\}\right]
$$

The joint PDF for first passage times can therefore be easily constructed in terms of the parameters of the joint PDF for a number of level crossings. For example, the joint PDF of first passage time $P_{T_{f_{1}} T_{f_{2}}((0,0)}\left(t_{1}, t_{2}\right)$ for a bivariate random process, under the assumption that $P\left[T_{f_{i}}=0\right]=0$, for $(i=1,2)$, is given by

$$
\begin{aligned}
P_{T_{f_{1}} T_{f_{2}} \mid(0,0)}\left(t_{1}, t_{2}\right)= & 1+\exp \left(-\left\{\left\langle N_{1}\left(\alpha_{1}, 0, t_{1}\right)\right\rangle+\left\langle N_{2}\left(\alpha_{2}, 0, t_{2}\right)\right\rangle\right.\right. \\
& \left.\left.-\operatorname{Cov}\left[N_{1}\left(\alpha_{1}, 0, t_{1}\right), N_{2}\left(\alpha_{2}, 0, t_{2}\right)\right]\right\}\right) \\
& -\exp \left[-\left\langle N_{1}\left(\alpha_{1}, 0, t_{1}\right)\right\rangle\right]-\exp \left[-\left\langle N_{2}\left(\alpha_{2}, 0, t_{2}\right)\right\rangle\right]
\end{aligned}
$$

If the conditions $P\left[T_{f_{i}}=0\right]=0(i=1,2)$ are not satisfied, Eq. (16) is modified as

$$
\begin{aligned}
& P\left\{\left[T_{f_{1}}\left(\alpha_{1}\right)<t_{1}\right] \cap\left[T_{f_{2}}\left(\alpha_{2}\right)<t_{2}\right]\right\} \\
& \quad=P_{T_{f_{1}} T_{f_{2}}}(0,0)+\left\{1-P_{T_{f_{1}} T_{f_{2}}}(0,0)\right\} P_{T_{f_{1}} T_{f_{2}} \mid(0,0)}\left(t_{1}, t_{2}\right)
\end{aligned}
$$

The procedure of computations for $\left\langle N_{1}\left(\alpha_{1}, 0, t_{1}\right)\right\rangle,\left\langle N_{2}\left(\alpha_{2}, 0, t_{2}\right)\right\rangle$, and $\operatorname{Cov}\left[N_{1}\left(\alpha_{1}, 0, t_{1}\right), N_{2}\left(\alpha_{2}, 0, t_{2}\right)\right]$ is essentially the same as outlined in Appendix I. A modification in the formulation is, however, required as $t_{1} \neq t_{2}$, the details of which have been worked out in Appendix II. The joint PDF for first passage times can be constructed for $k>2$ following similar arguments outlined in this section.

The marginal distribution for first passage time is given by $P_{T_{f_{1}}}\left(t_{1}\right)=P_{T_{f_{1}} T_{f_{2}}}\left(t_{1}, \infty\right)$. When $t_{2}=\infty$, for a given level $\alpha_{2}$, there are infinite number of crossings of $X_{2}(t)$, from which it follows that $\left\langle N_{2}\left(\alpha_{2}, 0, t_{2}\right)\right\rangle=\infty$. Thus, from Eq. (16), we get

$$
\begin{aligned}
P_{T_{f_{1}} T_{2}}\left(t_{1}, \infty\right)= & 1+\exp \left[-\left\langle N_{1}\left(\alpha_{1}, 0, t_{1}\right)\right\rangle-\infty+c\right] \\
& -\exp \left[-\left\langle N_{1}\left(\alpha_{1}, 0, t_{1}\right)\right\rangle\right]-\exp [-\infty] \\
= & 1-\exp \left[-\left\langle N_{1}\left(\alpha_{1}, 0, t_{1}\right)\right\rangle\right],
\end{aligned}
$$

where $c=\operatorname{Cov}\left[N_{1}\left(\alpha_{1}, 0, t_{1}\right), N_{2}\left(\alpha_{2}, 0, t_{2}\right)\right]$. The marginal distribu- 
tion function, $P_{T_{f_{1}}}\left(t_{1}\right)$, is therefore seen to be exponential, which conforms to the existing studies in the literature.

\section{Approximation for Extreme Value Distribution}

Extreme values of random processes, defined as $X_{m_{i}}$ $=\max _{0<t<t_{i}} X_{i}(t)(i=1, \ldots, k)$, are random variables and their joint probability distribution function is related to their first passage times through the relation

$$
P\left[\bigcap_{i=1}^{k}\left(X_{m_{i}} \leqslant \alpha_{i}\right)\right]=P\left\{\bigcap_{i=1}^{k}\left[T_{f_{i}}\left(\alpha_{i}\right)>t_{i}\right]\right\}
$$

where $t_{i}=T$ for $(i=1, \ldots, k)$. Using Eqs. (15) and (19), the joint extreme value distribution function can be expressed in terms of the probability distributions of zero crossings of their respective levels $\alpha_{i}$, for a vector of random processes. Thus for a $k$-dimensional vector of Gaussian random processes $\left\{X_{i}(t)\right\}_{i=1}^{k}$, the joint probability distribution of extremes is given by

$$
P_{X_{m_{1}} \cdots X_{m_{k}}}\left(\alpha_{1}, \ldots, \alpha_{k}\right)=\exp \left[-\sum_{i=1}^{k(k+1) / 2} \lambda_{i}\right]
$$

where $\left\{\lambda_{i}\right\}_{i=1}^{k(k+1) / 2}$ are the parameters of $C_{1}^{k}+C_{2}^{k}=k(k+1) / 2$ mutually independent Poisson random variables $\left\{U_{i}\right\}_{i=1}^{k(k+1) / 2}$.

The marginal extreme value distribution function is related to the joint distribution through the relation $P_{X_{m_{i}}}\left(\alpha_{i}\right)$ $=P_{X_{m_{1}} \cdots X_{m_{i}} \cdots X_{m_{k}}}\left(\infty, \ldots, \alpha_{i}, \ldots, \infty\right)$. Thus, for a bivariate extreme value distribution, the marginal distribution is given by $P_{X_{m_{1}}}\left(\alpha_{1}\right)=P_{X_{m_{1}} X_{m_{2}}}\left(\alpha_{1}, \infty\right)$. For threshold $\alpha_{2}=\infty$, the number of level crossings for $X_{2}(t)$ is zero, from which it follows that $\left\langle N_{2}\left(\infty, 0, t_{2}\right)\right\rangle=0$ and $\operatorname{Cov}\left[N_{1}\left(\alpha_{1}, 0, t_{1}\right), N_{2}\left(\infty, 0, t_{2}\right)\right]=0$. The expression for the bivariate extreme value distribution, in terms of the statistics of $N_{i}\left(\alpha_{i}, 0, t_{i}\right)$, is obtained from Eqs. (20) and (2), and substituting $\alpha_{2}=\infty$, we get

$$
\begin{aligned}
P_{X_{m_{1}} X_{m_{2}}}\left(\alpha_{1}, \infty\right)= & \exp \left\{-\left\langle N_{1}\left(\alpha_{1}, 0, t_{1}\right)\right\rangle-\left\langle N_{2}\left(\alpha_{2}, 0, t_{2}\right)\right\rangle\right. \\
& \left.+\operatorname{Cov}\left[N_{1}\left(\alpha_{1}, 0, t_{1}\right), N_{2}\left(\infty, 0, t_{2}\right)\right]\right\} \\
= & \exp \left[-\left\langle N_{1}\left(\alpha_{1}, 0, t_{1}\right)\right\rangle\right]
\end{aligned}
$$

The above result, along with Eq. (4), shows that the marginal distribution $P_{X_{m_{1}}}\left(\alpha_{1}\right)$ is Gumbel, which conforms to the existing results in random vibration literature. This also satisfies the requirement for multivariate extreme value distribution functions which specifies that the marginals should be nondegenerate (Kotz and Nadarajah 2000).

\section{Numerical Examples}

Two numerical examples are considered to illustrate the salient features of the proposed model. A detailed parametric study is first carried out on two mutually correlated, stationary, Gaussian random processes in order to study the effect of correlation of the parent processes on the joint distribution functions for first passage time and extreme values. The second example deals with the response analysis of a two-span bridge subjected to spatially varying random earthquake support motions. The motivation for this study is to demonstrate the effectiveness of the proposed theory in reliability calculations for real life engineering structures. The

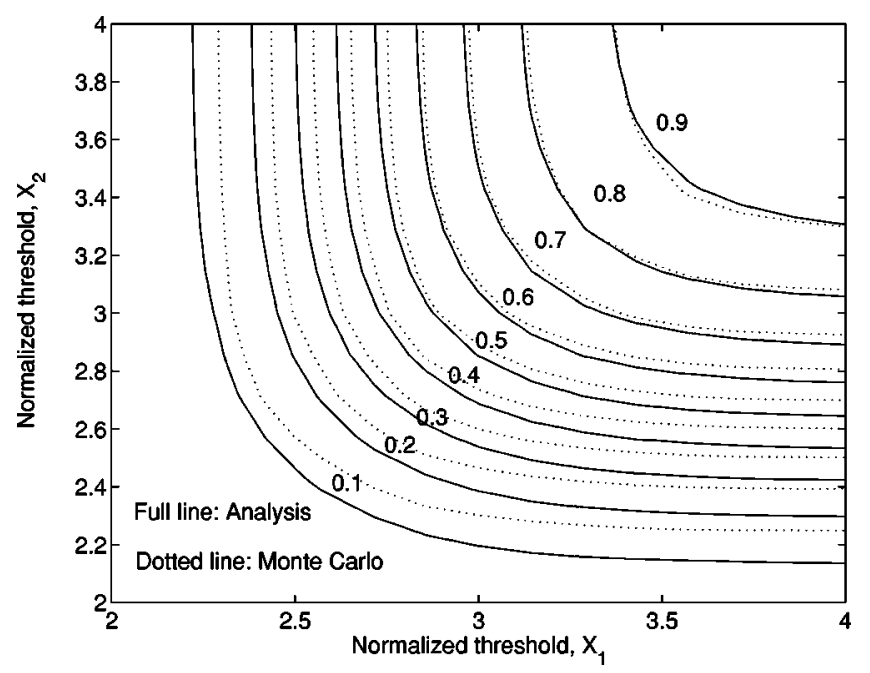

Fig. 1. Level curves of $P_{X_{m_{1}}} X_{m_{2}}\left(x_{1}, x_{2}\right)$ for the bivariate stationary, Gaussian random process (Example 1), $c=0.50$; the numbers on the figure indicate probability levels

analytical results for the two examples are compared with those obtained from full scale Monte Carlo simulations.

\section{Example 1: A Two-Dimensional Vector Random Process}

Consider two jointly stationary, zero mean, Gaussian random processes $X_{1}(t)$ and $X_{2}(t)$ with specified autocorrelation functions

$$
\begin{aligned}
& R_{11}(\tau)=S_{1}^{2} \exp \left[-\alpha \tau^{2}\right] \\
& R_{22}(\tau)=S_{2}^{2} \exp \left[-\beta \tau^{2}\right]
\end{aligned}
$$

respectively, with $\alpha, \beta>0$. The auto-PSD of $X_{1}(t)$ and $X_{2}(t)$ are obtained from the Wiener-Khintchine relations and are denoted by $S_{11}(\omega)$ and $S_{22}(\omega)$. The cross-PSD $S_{12}(\omega)$ is given by

$$
S_{12}(\omega)=c(\omega)\left|S_{11}(\omega) S_{22}(\omega)\right|^{0.5} \exp [i \gamma(\omega)]
$$

where $\gamma(\omega)=$ phase spectrum and $c(\omega)=$ coherence spectrum, which takes values in $[0,1]$. We assume $c(\omega)$ to have a constant value $c$, such that $0 \leqslant c \leqslant 1$, for all $\omega$ and $\gamma(\omega)=\omega / \psi$ with $\psi=8$. Clearly, if $c=0, X_{1}(t)$ and $X_{2}(t)$ are independent and, if, $c=1.0$, they are fully coherent. $\alpha$ and $\beta$ are taken to be 100 and 150 , respectively, and $S_{1}$ and $S_{2}$ are assumed to be equal to $\sqrt{2}$. The bandwidth of the processes $X_{1}(t)$ and $X_{2}(t)$, measured in terms of the spectral parameter, $q=\left[1-\lambda_{j_{1}}^{2} /\left(\lambda_{j_{0}} \lambda_{j_{2}}\right)\right]^{0.5}$ (Vanmarcke 1972), are, respectively, 0.6030 and 0.6032 . It must be noted that $q$ takes values in $[0,1]$, with small values denoting narrow-band processes and relatively large values denoting wide-band processes. The cross-correlation function is the Fourier transform of $S_{12}(\omega)$ and is shown to be of the form

$$
\begin{aligned}
R_{12}(\tau)= & \sqrt{2} c S_{1} S_{2} \exp \left\{-2 \alpha \beta(\tau \psi-1)^{2} /\right. \\
& {\left.\left[(\alpha+\beta) \psi^{2}\right]\right\} /\left\{(\alpha \beta)^{0.25}(1 / \alpha+1 / \beta)^{0.5}\right\} }
\end{aligned}
$$

The effect of coherence on the bivariate extreme value distribution is studied by assuming $c$ to have values equal to $0.05,0.50$, and 0.95 , respectively. Contour plots of $P_{X_{m_{1}} X_{m_{2}}}\left(x_{1}, x_{2}\right)$, that is, plots of the relation between $x_{1}$ and $x_{2}$, such that $P_{X_{m_{1}} X_{m_{2}}}\left(x_{1}, x_{2}\right)$ $=p$, for different values of $p$, are shown in Fig. 1 for the bivariate extreme value distribution of $X_{1}(t)$ and $X_{2}(t)$, when $c=0.50$ and 


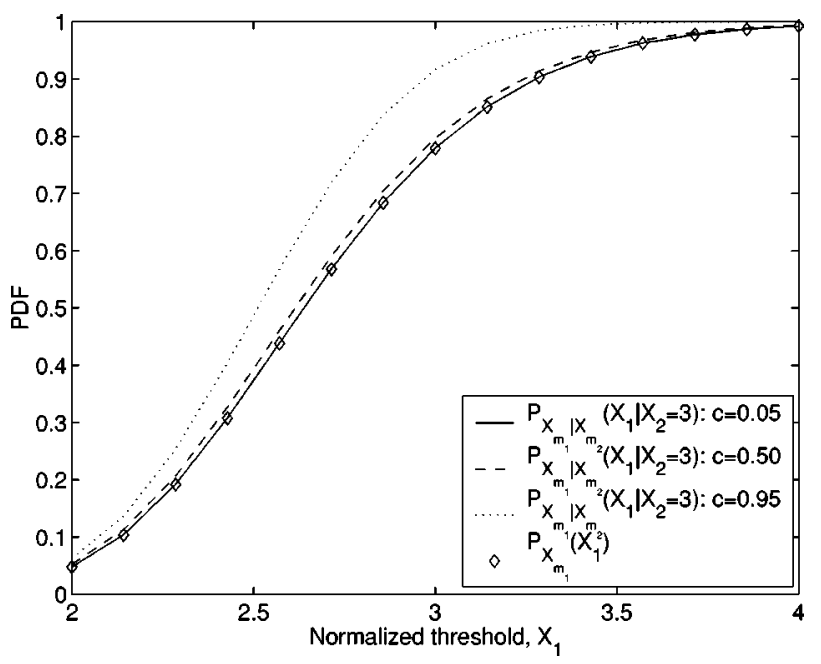

Fig. 2. Comparison of the conditional probability distribution function (PDF) $P_{X_{m_{1}} \mid X_{m_{2}}}\left(x_{1} \mid X_{m_{2}}=3\right)$ with the univariate PDF $P_{X_{m_{1}}}\left(x_{1}\right)$

$T=10 \mathrm{~s}$. Henceforth in this paper, the plots of this type are referred to as level curves. The analytical predictions are validated with those obtained from Monte Carlo simulations. Based on the known PSD matrix of $X_{1}(t)$ and $X_{2}(t)$, an ensemble of 5,000 samples for the vector stationary, Gaussian processes are digitally simulated using the well-known spectral decomposition method. From each of these samples, the extremes of $X_{1}(t)$ and $X_{2}(t)$, over the specified duration, are detected. The resulting ensemble of extreme values is processed subsequently to estimate the joint PDF. The analytical results are observed to be in fairly good agreement with those obtained from Monte Carlo simulations, especially in the higher end of the probability distribution function. This is to be expected since the assumption of Poisson crossing is more realistic toward the tails of the probability distribution functions. The joint PDF $P_{X_{m_{1}} X_{m_{2}}}\left(x_{1}, x_{2}\right)$ was found to be significantly dependent on the parameter $c$; thus, for instance, the value of $P_{X_{m_{1}} X_{m_{2}}}(3,3)$ for $c=0.05,0.050$, and 0.95 , was observed to be $0.5614,0.5638$, and 0.6542 ; the corresponding results from the Monte Carlo simulations were $0.5737,0.5867$, and 0.6748. A comparison of the conditional distribution $P_{X_{m_{1}} X_{m_{2}}}\left(x_{1} \mid X_{m_{2}}=x_{2}\right)$ with the univariate probability distribution function, $P_{X_{m_{1}}}\left(x_{1}\right)$, is shown in Fig. 2, when $X_{m_{2}}=3$ and for various values of $c$. As the coherence between the parent random processes weaken, the conditional distribution is observed to approach the univariate PDF, as expected, and the differences almost vanish when $c=0.05$. Similar plots were constructed for $X_{m_{2}}=2.0$ and $X_{m_{2}}=2.5$ and an examination of these figures (not shown here) revealed that the impact of linear dependence is negligible for values of $c<0.5$, indicating that the dependence is unimportant unless the processes are highly coherent. It is of interest to note that Ditlevsen (1979), in his studies on the series system reliability of structures under random static loads, observed a similar insensitivity on the system reliability when the correlation coefficients between the performance functions of the various failure modes were below 0.5 .

Similar studies on the bivariate distribution function for first passage times have also been carried out. Fig. 3 illustrates the level curves for the bivariate PDF for first passage times $P_{T_{f_{1}} T_{f_{2}}}\left(t_{1}, t_{2}\right)$ when $c=0.95$ and the normalized thresholds $x_{1}$ and $x_{2}$ are taken to 3 and 2 , respectively. The analytical predictions

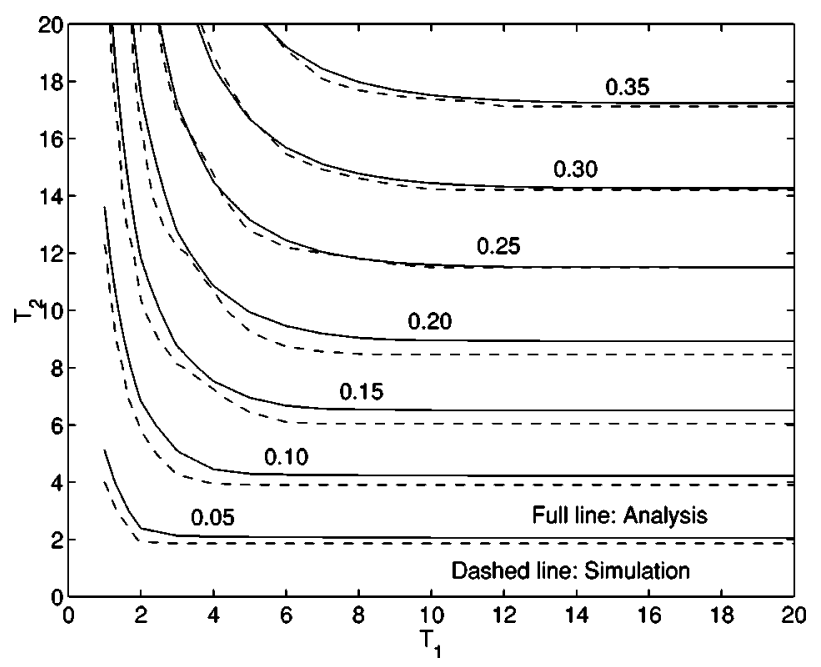

Fig. 3. Level curves for the bivariate first passage time $P_{T_{f_{1}} T_{f_{2}}}\left(t_{1}, t_{2}\right)$ for bivariate stationary, Gaussian random processes for $c=0.95$ and normalized thresholds $x_{1}=3$ and $x_{2}=2$ (Example 1); the numbers on the figure indicate probability levels

are compared with those obtained from Monte Carlo simulations. Here, the spectral decomposition method is once again used to digitally simulate an ensemble of 5,000 samples of the time histories. In each of these samples, the time length of the sample is increased until the specified threshold is crossed for the first time. The resulting ensemble of first passage times, so obtained, is processed subsequently to estimate the joint PDF of $T_{f_{1}}$ and $T_{f_{2}}$. The number of terms, $N_{s}$, considered in the spectral expansion in the simulation procedure depends on the time length of the process, $T$, and the spectral bandwidth $\delta_{\omega}=\left(\omega_{u}-\omega_{l}\right)$ and is given by $N_{s}$ $=\Delta_{\omega} T / 2 \pi$. In this example, we consider $\omega_{u}=0$ and $\omega_{l}=30 \mathrm{~Hz}$. The close agreement that is observed to exist between the predictions serves to illustrate the acceptability of the proposed model. The conditional distribution for first passage time $P_{T_{f_{1} \mid T_{f_{2}}}}\left(t_{1} \mid T_{f_{2}}\right.$ $=6)$ is compared with the univariate PDF for first passage time when $c=0.95$ in Fig. 4. Once again, a good agreement is observed

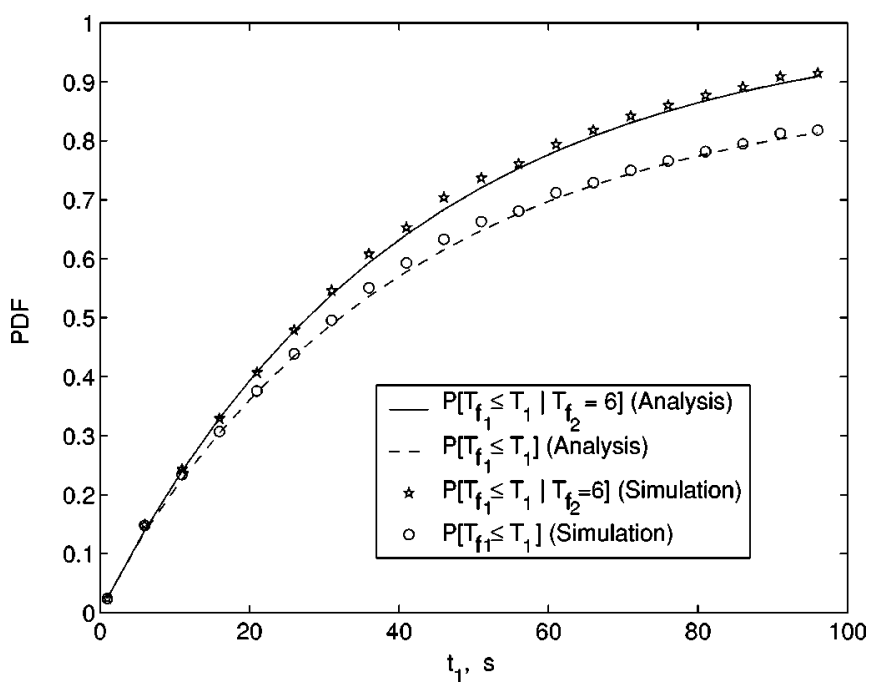

Fig. 4. Comparison of the conditional probability distribution function (PDF) for first passage time $P_{T_{f_{1}} \mid T_{f_{2}}}\left(t_{1} \mid T_{f_{2}}=6\right)$ and the univariate PDF $P_{T_{f_{1}}}\left(t_{1}\right)$; (Example 1) 
between the analytical and simulation results. The difference in the probability levels between the conditional distribution and the univariate PDF can be attributed to the coherence between the parent random processes.

The applicability of the proposed bivariate Poisson model used in this example is checked for the condition given in Eq. (14). For $c=0.05, \lambda_{1}, \lambda_{2}$, and $\lambda_{3}$ are $0.0075,0.0092$, and $1.1776 \times 10^{-6}$, respectively, and correspondingly, $\operatorname{corr}\left[N_{1}, N_{2}\right]=1.4093 \times 10^{-4}$ is less than the upper bound $1.5597 \times 10^{-4}$. For $c=0.50, \lambda_{1}$ $=0.0074, \lambda_{2}=0.0091, \lambda_{3}=1.8076 \times 10^{-4}$, the upper bound is 0.0239 and $\operatorname{corr}\left[N_{1}, N_{2}\right]=0.0216$. Similarly, for $c=0.95$, the three parameters $\left\{\lambda_{i}\right\}_{i=1}^{3}$ are, respectively, 0.0035, 0.0052, and 0.0040, $\operatorname{corr}\left[N_{1}, N_{2}\right]=0.4802$ which is less than the bound 0.5314 . These observations conform to the expectation that, in our study, Eq. (14) is always satisfied since the study employs an inverse approach to determine parameters $\left\{\lambda_{i}\right\}_{i=1}^{3}$.

\section{Example 2: Two Span Bridge under Seismic Excitations}

A two-span bridge, idealized as a two-span Euler-Bernoulli beam with three supports, with the beams assumed to be continuous over the interior support, is considered. The structure is taken to have a constant mass per unit length $m$ and flexural rigidity $E I$, such that $E I / m=40.50 \times 10^{6} \mathrm{~m}^{4} / \mathrm{s}^{2}$. The spans are assumed to be of length 55 and $45 \mathrm{~m}$, respectively. The structure is discretized into 40, two-noded beam elements, with one translational and one rotational degree of freedom per node. A free vibration analysis revealed that the first five natural frequencies of the structure are $3.79,6.70,14.68,22.37$, and $32.49 \mathrm{~Hz}$, respectively.

The bridge is assumed to be subjected to spatially varying earthquake accelerations with zero mean. Only the transverse component of the earthquake accelerations at the three supports are considered in the analysis and is specified through a $6 \times 6$ PSD matrix. The auto-PSD functions for the accelerations at the $i$ th support, $S_{i i}$, are assumed to be of the form

$$
S_{i i}(\omega)=S_{0_{i}} \Omega_{i}^{2}\left(\Omega_{i}+4 \eta_{i}^{2} \omega^{2}\right) /\left\{\left(\Omega_{i}^{2}-\omega^{2}\right)^{2}+4\left(\eta_{i} \Omega_{i} \omega\right)^{2}\right\}
$$

where $S_{0}=$ measure of the intensity of the ground acceleration and $\Omega_{i}$ and $\eta_{i}$ are parameters dependent on the local soil conditions at the $i$ th support. It is assumed that $\left(\Omega_{1}, \Omega_{2}, \Omega_{3}\right)$ $=(1.72,1.59,1.52) \mathrm{Hz}$ and $\left(\eta_{1}, \eta_{2}, \eta_{3}\right)=(0.60,0.55,0.50)$. The accelerations at all the supports are assumed to have the same measure of intensity $S_{0_{i}}=0.014 \mathrm{~m}^{2} / \mathrm{s}^{4}(i=1,2,3)$. The coherency function of the ground accelerations between any two supports $j$, $l, j \neq l(j, l=1,2,3)$ is of the form (Der Kiureghian 1996)

$$
\gamma_{j l}(\omega)=\left|\gamma_{j l}(\omega)\right| \exp \left[i \Phi_{j l}(\omega)\right]
$$

and in the present study, the parameters $\left|\gamma_{j l}(\omega)\right|$ and $\Phi_{j l}(\omega)$ are modeled as per Abrahamson et al. (1991) and are given by

$$
\left|\gamma_{j l}(\omega)\right|=\tanh \left\{\left(a_{1}+a_{2} D_{j l}\right)\left[\exp \left\{\left(b_{1}+b_{2} D_{j l}\right) f\right\}+1 / 3 f^{c}\right]+0.35\right\}
$$

$$
\Phi_{j l}(\omega)=-i \log \left\{h_{j l}(\omega) \exp \left[i \phi_{j l}(\omega)\right]+\left[1-h_{j l}(\omega)\right] \exp [i \theta(\omega)]\right\}
$$

where $\quad \phi_{j l}(\omega)=\omega D_{j l} / V, \quad h_{j l}(\omega)=\left\{1+(f / 19)^{4}\right\}^{-1}, \quad a_{1}=2.535, \quad a_{2}$ $=-0.0118, b_{1}=-0.115, b_{2}=-8.37 \times 10^{-4}, c=-0.878, V=500 \mathrm{~m} / \mathrm{s}$, and $D_{j l}$ is the distance between supports $j$ and $l$.

The structure is analyzed separately for the dynamic and pseudostatic components of the midpoint displacements at the two spans. The safety of the bridge structure is assumed to be defined through the dynamic components of the midpoint displacements,

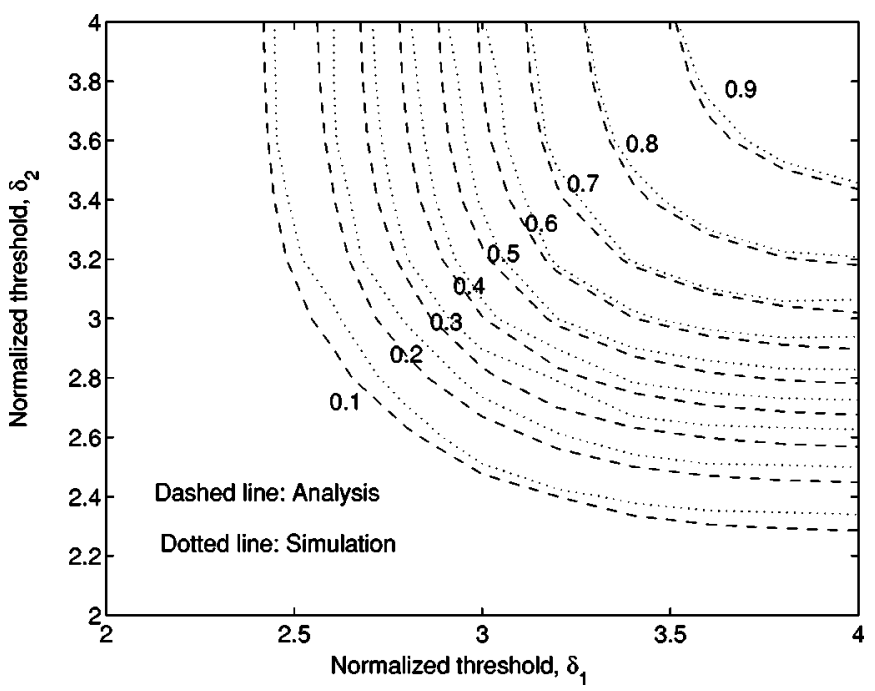

Fig. 5. Level curves for $P_{\Delta_{m_{1}} \Delta_{m_{2}}}\left(\delta_{1}, \delta_{2}\right)$ for stationary, Gaussian random processes $\Delta_{1}(t)$ and $\Delta_{2}(t)$ (Example 2); the numbers on the figure indicate probability levels

$\Delta_{1}(t)$ and $\Delta_{2}(t)$, respectively. For the dynamic analysis, the damping is assumed to be viscous and proportional. The mass and stiffness proportionality constants are adjusted such that the damping is $5 \%$ in the first two modes and are, respectively, taken to be $0.4939 \mathrm{~s}^{-1}$ and $2.9960 \times 10^{-5} \mathrm{~s}$. Since the excitations at the supports are stationary, Gaussian random processes and linear structural behavior are assumed, $\Delta_{1}(t)$ and $\Delta_{2}(t)$, for large $t$, are also jointly stationary, Gaussian random processes whose autoPSD functions $\left[S_{\Delta_{1} \Delta_{1}}(\omega)\right.$ and $\left.S_{\Delta_{2} \Delta_{2}}(\omega)\right]$ and cross-PSD function $\left[S_{\Delta_{1} \Delta_{2}}(\omega)\right]$ are obtained as linear combinations of $S_{i j}(i, j$ $=1,2,3)$. The coherence spectrum for $\Delta_{1}$ and $\Delta_{2}$ is expressed as $|\gamma(\omega)|=S_{\Delta_{1} \Delta_{2}}(\omega) /\left[S_{\Delta_{1} \Delta_{1}}(\omega) S_{\Delta_{2} \Delta_{2}}(\omega)\right]^{0.5}$ and was observed to take values over a range of about $0.2-1.0$, as frequency was varied over $0-40 \mathrm{~Hz}$. The spectral shape factors, $q$, for $\Delta_{1}(t)$ and $\Delta_{2}(t)$ are, respectively, 0.3216 and 0.3424 . The threshold displacements for the random processes $\Delta_{1}$ and $\Delta_{2}$ are normalized with respect to their respective standard deviations and the normalized thresholds are varied from 2 to 4 . The level curves for the bivariate extreme value distributions $P_{\Delta_{m_{1}} \Delta_{m_{2}}}\left(\delta_{1}, \delta_{2}\right)$ obtained from the analysis are shown in Fig. 5. The extreme value distributions were determined for time durations $T=9 \mathrm{~s}$. The procedure to obtain results from Monte Carlo simulations is the same as that used in Example 1. Here, 1,000 samples of time histories of $\Delta_{1}(t)$ and $\Delta_{2}(t)$ are digitally simulated based on the knowledge of a PSD matrix of $\Delta_{1}(t)$ and $\Delta_{2}(t)$. This leads to an ensemble of 1,000 realizations of extremes and the first passage times, which, in turn, are processed to estimate the desired joint PDFs. The level curves for the extreme value distributions obtained from the analysis are observed to bear a close resemblance to those obtained from Monte Carlo simulations. The parameters considered in using the spectral decomposition method for simulating time histories are $\omega_{l}=0.1 \mathrm{rad} / \mathrm{s}, \omega_{u}=250 \mathrm{rad} / \mathrm{s}$, and $N_{s}=358$. As expected, the agreement is observed to be better at the higher end of the joint distribution function where the assumption of Poisson crossings is more realistic. Fig. 6 compares the conditional PDF $P_{\Delta_{m_{1}} \Delta_{m_{2}}}\left(\delta_{1} \mid \Delta_{m_{2}}=\delta_{2}\right)$ with the univariate PDF $P_{\Delta_{m_{1}}}\left(\delta_{1}\right)$ for normalized threshold $\delta_{2}=3.2$. The analytical predictions can be seen to be in close agreement with those obtained from Monte Carlo simulations on 1,000 samples, especially at higher threshold lev- 


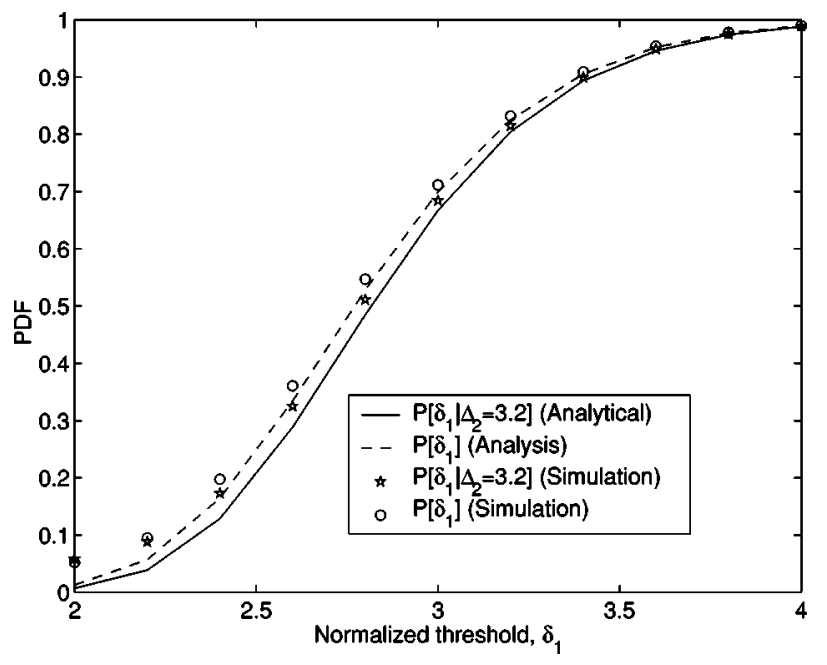

Fig. 6. Comparison of the conditional probability distribution function (PDF) $P_{\Delta_{m_{1}} \mid \Delta_{m_{2}}}\left(\delta_{1} \mid \Delta_{m_{2}}=3.2\right)$ and the univariate PDF $P_{\Delta_{m_{1}}}\left(\delta_{1}\right)$ (Example 2)

els. The difference in the probability levels between the conditional PDF and the univariate PDF is again due to the coherence between the parent random processes $\Delta_{1}(t)$ and $\Delta_{2}(t)$. The bivariate PDF for first passage times, $P_{T_{f_{1}} T_{f_{2}}}\left(t_{1}, t_{2}\right)$, is constructed for $0 \leqslant t_{1}, t_{2} \leqslant 40 \mathrm{~s}$. The normalized thresholds $\delta_{1}$ and $\delta_{2}$ were taken to be 3 and 2, respectively. The level curves for the joint PDF, $P_{T_{f_{1}} T_{f_{2}}}\left(t_{1}, t_{2}\right)$ obtained from both analysis as well as simulations carried out on 1,000 samples, are shown in Fig. 7. It is observed that the analytical predictions are in close agreement with the predictions based on Monte Carlo simulations at higher threshold levels. For the bivariate Poisson distribution used in this example, the parameters $\lambda_{1}, \lambda_{2}$, and $\lambda_{3}$, respectively, turn out to be 0.0108 , 0.0145 , and $2.8506 \times 10^{-5}$. Using these values in Eq. (14), we get $\operatorname{corr}\left[N_{1}, N_{2}\right]=0.0023$ and the upper bound is 0.0026 . Thus, as in

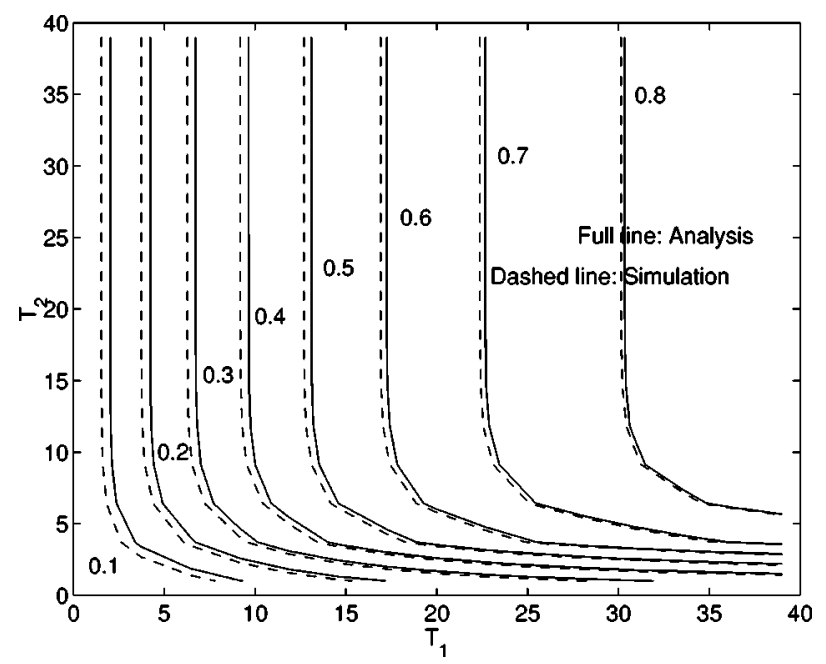

Fig. 7. Level curves for the joint probability distribution function for first passage time $P_{T_{f_{1}} T_{f_{2}}}\left(t_{1}, t_{2}\right)$ for the two stationary, Gaussian random processes, $\Delta_{1}$ and $\Delta_{2}$ (Example 2); the numbers on the figure indicate probability levels the previous example, we see that the inverse approach adopted in this study to determine the parameters $\lambda_{i}$ always satisfies the condition given in Eq. (14).

\section{Conclusions}

Approximations for the joint probability distribution functions for the number of level crossings, the first passage times, and the extreme values associated with a vector of mutually correlated, stationary, Gaussian random processes have been developed. The proposed approximations predict that for mutually correlated parent processes, their respective number of level crossings, their first passage times, and their extreme values are also correlated. These predictions have been validated by Monte Carlo simulations carried out on the examples considered in this paper. The multivariate extreme value distribution inherits the inaccuracies associated with the marginal distributions, as reported in the literature, when level crossings are modeled as Poisson random variables. The marginal distributions derived from the joint probability distribution functions for first passage times and extreme values are found to be exponential and Gumbel, respectively, and these confirm the existing studies in the literature on scalar random processes. Existing studies on outcrossings involving the vector random process essentially formulate the problem as an outcrossing of a scalar random process. In this context, the proposed method can be viewed as an alternative approach where the joint outcrossings of vector random processes have been studied. The proposed multivariate extreme value distribution functions have applications in reliability analyses of structural systems with multiple limit states. Further work involving extension of the proposed method to problems involving nonstationary and nonGaussian random processes is currently being pursued by the present writers.

\section{Acknowledgment}

The work reported in this paper forms a part of the research project entitled "Seismic probabilistic safety assessment of nuclear power plant structures," funded by the Board of Research in Nuclear Sciences, Department of Atomic Energy, Government of India.

\section{Appendix I}

$\left\langle N_{1}\left(\alpha_{1}, 0, T\right) N_{2}\left(\alpha_{2}, 0, T\right)\right\rangle$ is obtained as a six-dimensional integral and if only level crossings with positive slopes are considered, one gets

$$
\begin{aligned}
\left\langle N_{1}^{+} N_{2}^{+}\right\rangle= & \int_{0}^{T} \int_{0}^{T} \int_{-\infty}^{\infty} \int_{-\infty}^{\infty} \int_{0}^{\infty} \int_{0}^{\infty}\left\{\dot{x}_{1} \dot{x}_{2} \delta\left[x_{1}-\alpha_{1}\right] \delta\left[x_{2}-\alpha_{2}\right]\right. \\
& \left.\times p_{X_{1} X_{2} \dot{X}_{1} \dot{X}_{2}}\left(x_{1}, x_{2}, \dot{x}_{1}, \dot{x}_{2} ; t_{1}, t_{2}\right)\right\} d x_{1} d x_{2} d \dot{x}_{1} d \dot{x}_{2} d t_{1} d t_{2}
\end{aligned}
$$

Here, $N_{i}^{+}$denotes crossing of level $\alpha$ with positive slope. The inner four integrals can be simplified and written as 


$$
I\left(t_{1}, t_{2}\right)=\int_{0}^{\infty} \int_{0}^{\infty} \dot{x}_{1} \dot{x}_{2} p_{X_{1} X_{2} \dot{X}_{1} \dot{X}_{2}}\left(\alpha_{1}, \alpha_{2}, \dot{x}_{1}, \dot{x}_{2} ; t_{1}, t_{2}\right) d \dot{x}_{1} d \dot{x}_{2}
$$

For stationary random processes $X_{1}\left(t_{1}\right)$ and $X_{2}\left(t_{2}\right), I\left(t_{1}, t_{2}\right)=I\left(t_{2}\right.$ $\left.-t_{1}\right)$. Substituting $\tau=t_{2}-t_{1}$ in Eq. (30) leads to the simplification

$$
\left\langle N_{1}^{+} N_{2}^{+}\right\rangle=\int_{-T}^{T}(T-|\tau|) I(\tau) d \tau
$$

If $X_{1}(t)$ and $X_{2}(t)$ are assumed to be stationary Gaussian random processes, the four-dimensional joint pdf $p_{X_{1} X_{2} \dot{X}_{1} \dot{X}_{2}}$ $\left(\alpha_{1}, \alpha_{2}, \dot{x}_{1}, \dot{x}_{2} ; t_{1}, t_{2}\right)$ is also Gaussian and is of the form

$$
p_{X_{1} X_{2} \dot{X}_{1} \dot{X}_{2}}\left(\alpha_{1}, \alpha_{2}, \dot{x}_{1}, \dot{x}_{2} ; t_{1}, t_{2}\right)=1 /\left(4 \pi^{2}|\Delta|^{0.5}\right) \exp \left[-0.5\left\{Z^{\prime} \Delta^{-1} Z\right\}\right]
$$

where $Z^{\prime}=\left[\alpha_{1} \alpha_{2} \dot{x}_{1} \dot{x}_{2}\right]^{\prime}$ and

$$
\Delta=\left[\begin{array}{cccc}
s_{1} & r(\tau) & 0 & r^{\prime}(\tau) \\
r(\tau) & s_{2} & -r^{\prime}(\tau) & 0 \\
0 & -r^{\prime}(\tau) & m_{1} & -r^{\prime \prime}(\tau) \\
r^{\prime}(\tau) & 0 & -r^{\prime \prime}(\tau) & m_{2}
\end{array}\right]
$$

Here, $s_{1}=\left\langle X_{1}^{2}\left(t_{1}\right)\right\rangle, s_{2}=\left\langle X_{2}^{2}\left(t_{2}\right)\right\rangle, m_{1}=\left\langle\dot{X}_{1}^{2}\left(t_{1}\right)\right\rangle, m_{2}=\left\langle\dot{X}_{2}^{2}\left(t_{2}\right)\right\rangle, \quad r(\tau)$ $=\left\langle X_{1}\left(t_{1}\right) X_{2}\left(t_{2}\right)\right\rangle$, and $\left\langle\partial X_{1}^{m}\left(t_{1}\right) / \partial t_{1}^{m} \partial X_{2}^{n}\left(t_{2}\right) / \partial t_{2}^{n}\right\rangle=(-1)^{m} \partial r^{m+n} / \partial \tau^{m+n}$. Using arguments provided in Cramer and Leadbetter (1967), Eq. (32) can be simplified to get

$$
\begin{aligned}
& p_{X_{1} X_{2} \dot{X}_{1} \dot{X}_{2}}\left(\alpha_{1}, \alpha_{2}, \dot{x}_{1}, \dot{x}_{2} ; t_{1}, t_{2}\right) \\
& \quad=1 /\left(4 \pi^{2}|\Delta|^{0.5}\right) \exp \left[-0.5\left(b_{0}+b_{1} \dot{x}_{1}+b_{2} \dot{x}_{2}+b_{3} \dot{x}_{1}^{2}\right.\right. \\
& \left.\left.\quad+b_{4} \dot{x}_{2}^{2}+b_{5} \dot{x}_{1} \dot{x}_{2}\right)\right],
\end{aligned}
$$

where

$$
\begin{gathered}
b_{0}=\left\{M_{11} \alpha_{1}^{2}+\left(M_{21}+M_{12}\right) \alpha_{1} \alpha_{2}+M_{22} \alpha_{2}^{2}\right\} /|\Delta| \\
b_{1}=\left\{\left(M_{31}+M_{13}\right) \alpha_{1}+\left(M_{32}+M_{23}\right) \alpha_{2}\right\} /|\Delta| \\
b_{2}=\left\{\left(M_{41}+M_{14}\right) \alpha_{1}+\left(M_{42}+M_{24}\right) \alpha_{2}\right\} /|\Delta| \\
b_{3}=M_{33} /|\Delta| \\
b_{4}=M_{44} /|\Delta| \\
b_{5}=\left\{M_{33}+M_{44}\right\} /|\Delta|
\end{gathered}
$$

$|\Delta|$ denotes the determinant of $\Delta$ and $M_{i j}(i, j=1, \ldots, 4)$ $=$ cofactors of $\Delta$. After substituting Eq. (34) in Eq. (32), the resulting integral was evaluated symbolically using MAPLE, to get

$$
\begin{aligned}
I(\tau)= & \exp \left[-b_{0} / 2\right] /\left(4 \Pi^{2}|\Delta|^{0.5}\right) \\
& \times \int_{0}^{\infty} \dot{x}_{2} I_{0}\left(\dot{x}_{2}\right) \exp \left[-0.5\left(b_{2} \dot{x}_{2}+b_{4} \dot{x}_{2}^{2}\right)\right] d \dot{x}_{2}
\end{aligned}
$$

where

$$
\begin{aligned}
I_{0}\left(\dot{x}_{2}\right)= & \left(1+0.6267 c\left(\dot{x}_{2}\right) \exp \left\{0.1250 c^{2}\left(\dot{x}_{2}\right)\right\}\right. \\
& \left.\times\left\{\operatorname{erf}\left[0.3536 c\left(\dot{x}_{2}\right)\right]-1\right\}\right) / b_{3}
\end{aligned}
$$

and $c\left(\dot{x}_{2}\right)=\left(b_{1}+b_{5} \dot{x}_{2}\right) /\left(b_{3}\right)^{0.5}$. Eq. (31), together with Eq. (36), thus leads to a double integral which needs to be evaluated numerically. Also, if the processes are uncorrelated, $r(\tau)=0$, and it can be shown that $p_{X_{1} X_{2} \dot{X}_{1} \dot{X}_{2}}\left(\alpha_{1}, \alpha_{2}, \dot{x}_{1}, \dot{x}_{2} ; t_{1}, t_{2}\right)$ in Eq. (32) can be replaced as $p_{X_{1}}\left(\alpha_{1}\right) p_{X_{2}}\left(\alpha_{2}\right) p_{\dot{X}_{1}}\left(\dot{x}_{1}\right) p_{\dot{X}_{2}}\left(\dot{x}_{2}\right)$. This facilitates a closed form solution of Eq. (29) and it can be shown that $\left\langle N_{1} N_{2}\right\rangle$ $=\left\langle N_{1}\right\rangle\left\langle N_{2}\right\rangle$, where $\left\langle N_{i}\right\rangle$ corresponds to the form given in Eq. (4).

When level crossings with negative slopes are also included, $N_{i}=N_{i}^{+}+N_{i}^{-}$. Here, $N_{i}^{-}$denotes the crossing of level $-\alpha$ with a negative slope. Thus $\left\langle N_{1} N_{2}\right\rangle=\left\langle N_{1}^{+} N_{2}^{+}\right\rangle+\left\langle N_{1}^{+} N_{2}^{-}\right\rangle+\left\langle N_{1}^{-} N_{2}^{+}\right\rangle+\left\langle N_{1}^{-} N_{2}^{-}\right\rangle$. The evaluation of each of these expectations requires the solution of a six-dimensional integration of the form given in Eq. (29). The domain of integration with respect to $\dot{x}_{i}$ depends on whether the level crossing considered is with a positive slope or negative slope and is either $[0,+\infty]$ or $[-\infty, 0]$. The joint pdf, given by Eq. (34), is an odd function with respect to $\dot{x}_{i}$, and hence four integrals of the form given by Eq. (29) need to be evaluated to determine $\left\langle N_{i} N_{j}\right\rangle$. On the other hand, for a scalar stationary Gaussian random process, the joint pdf $p_{X X} \dot{x}(x, \dot{x})$ is an even function in $\dot{x}$. Thus $\left\langle N_{i}^{+}\right\rangle=\left\langle N_{i}^{-}\right\rangle$, and $\left\langle N_{i}\right\rangle$ is obtained by multiplying the righthand side of Eq. (4) by a factor of 2 .

\section{Appendix II}

When $t_{1} \neq t_{2} \neq t$, the expression for $\left\langle N_{1}\left(\alpha_{1}, 0, t_{1}\right) N_{2}\left(\alpha_{2}, 0, t_{2}\right)\right\rangle$, as given in Eq. (31), needs to be modified. Following similar arguments as in deriving the expressions in Eq. (31), we get

$$
\begin{aligned}
\lambda_{N_{i} N_{j}}= & \int_{-t_{i}}^{t_{j}-t_{i}}\left\{t_{j}-|\tau|+\left|t_{j}-t_{i}\right|\right\} I(\tau) d \tau+\int_{t_{j}-t_{i}}^{0} t_{j} I(\tau) d \tau \\
& +\int_{0}^{t_{j}}\left(t_{i}-\tau\right) I(\tau) d \tau \quad \text { if } t_{j} \leqslant t_{i} \\
= & \int_{T_{i}}^{0}\left(t_{i}-|\tau|\right) I(\tau) d \tau+\int_{0}^{t_{j}-t_{i}} t_{i} I(\tau) d \tau \\
& +\int_{t_{j}-t_{i}}^{t_{j}}\left(t_{j}-\tau\right) I(\tau) d \tau \quad \text { if } t_{j}>t_{i}
\end{aligned}
$$

\section{References}

Abrahamson, N. A., Schneider, F. S., and Stepp, C. (1991). "Spatial coherency of shear waves from Lotung, Taiwan large-scale seismic test." Struct. Safety, 10, 145-162.

Castillo, E. (1988). Extreme value theory in engineering, Academic, Boston.

Cramer, H. (1966). "On the intersections between the trajectories of a normal stationary stochastic process and a high level." Ark. Mat., 6, 337-349.

Cramer, H., and Leadbetter, M. R. (1967). Stationary and related stochastic processes: Sample function properties and their applications, Wiley, New York.

Der Kiureghian, A. (1996). "A coherency model for spatially varying ground motions." Earthquake Eng. Struct. Dyn. 25(1), 99-111.

Ditlevsen, O. (1979). "Narrow reliability bounds for structural systems." J. Struct. Mech., 7(4), 453-472.

Ditlevsen, O. (1984). "First outcrossing probability bounds." J. Eng. Mech., 110(2), 282-292.

Galambos, J. (1978). The asymptotic theory of extreme order statistics, Wiley, New York.

Hagen, O. (1992). "Conditional and joint failure surface crossing of stochastic processes.” J. Eng. Mech., 118(9), 1814-1839.

Hagen, O., and Tvedt, L. (1991). "Vector process out-crossing as parallel 
system sensitivity measure." J. Eng. Mech., 117(10), 2201-2220.

Johnson, N. L., and Kotz, S. (1969). Discrete distributions, Wiley, New York.

Kotz, S., and Nadarajah, S. (2000). Extreme value distributions, Imperial College Press, London.

Leira, B. J. (1994). "Multivariate distributions of maxima and extremes for Gaussian vector processes." Struct. Safety 14, 247-265.

Leira, B. J. (2003). "Extremes of Gaussian and non-Gaussian vector processes: A geometric approach.” Struct. Safety, 25, 401-422.

Lin, Y. K. (1967). Probabilistic theory of structural dynamics, McGrawHill, New York.

Lutes, L. D., and Sarkani, S. (1997). Stochastic analysis of structural and mechanical vibrations, Prentice-Hall, Englewood Cliffs, N.J.

Madsen, H. O., Krenk, S., and Lind, N. C. (1986). Methods of structural safety, Prentice-Hall, Englewood Cliffs, N.J.

Melchers, R. E. (1999). Structural reliability analysis and prediction, Wiley, Chichester.

Naess, A. (1989). "A study of linear combination of load effects." $J$. Sound Vib., 129(2), 83-98.
Nigam, N. C. (1983). Introduction to random vibration, MIT Press, Cambridge, Mass.

Roberts, J. B. (1986). "First passage probabilities for randomly excited systems: diffusion methods." Probab. Eng. Mech. 1, 66-81.

Spencer, B. F., and Bergman, L. A. (1985). "The first passage problem in random vibration for a simple hysteretic oscillator." Technical Rep. AAE 85-8, University of Illinois, Urbana, Ill.

Srinivasan, S. K., and Mehata, K. M. (1976). Stochastic processes, TataMcGraw-Hill, New York.

Vanmarcke, E. H. (1972). "Properties of spectral moments with applications to random vibrations." J. Eng. Mech. Div., 98(2), 425-46.

Vanmarcke, E. H. (1975). "On the distribution of the first passage time for normal stationary random processes." J. Appl. Mech., 42, 215220.

Veneziano, D., Grigoriu, M., and Cornell, C. A. (1977). "Vector-process models for system reliability." J. Eng. Mech. Div., 103(3), 441-460.

Wen, Y. K., and Chen, H.-C. (1989). "System reliability under time varying loads: I.” J. Eng. Mech., 115(4), 808-823. 